\title{
El lenguaje de las predicciones meteorológicas. De los boletines y los productos a los medios de comunicación social
}

https://doi.org/10.31978/639-19-010-0.787

\author{
Delia Gutiérrez Rubio ${ }^{1}$ (dgutierrezr@aemet.es) \\ Ana Casals Carro' (acasalsc@aemet.es) \\ Rubén del Campo Hernández ${ }^{1}$ (rcampoh@aemet.es)
}

${ }^{1}$ AEMET / Área de Información Meteorológica y Climatológica

\begin{abstract}
RESUMEN
En esta ponencia proponemos una mirada sobre nuestros productos y nuestro léxico desde el punto de vista de la utilidad y la comprensibilidad para el usuario. Es decir, queremos hacer una revisión de la forma, más que del contenido, de nuestras predicciones; nos fijaremos en algunos de los productos o conceptos que más habitualmente suscitan dudas, controversias o gran expectación; pondremos algunos ejemplos de cómo manejamos en los medios y las redes sociales estas cuestiones, y haremos algunas propuestas para mejorar la comprensión de nuestras predicciones.
\end{abstract}

PALABRAS CLAVE: productos de predicción; léxico; orientación al usuario; redes sociales; medios de comunicación; comprensión de las predicciones.

\section{INTRODUCCIÓN}

Las predicciones meteorológicas se expresan mediante diferentes productos (boletines, mapas, gráficos, tablas, etc.), cada uno de los cuales implica un léxico, un formato, una estructura bien definidos y cuyo objetivo debe ser expresar con el mayor rigor a la vez que de manera comprensible para el usuario la información de la que disponemos.

Idealmente, los productos deben estar diseñados no para el consumo interno, o exclusivamente con nuestros criterios, sino a la medida de las necesidades y capacidades de los usuarios. Esto puede ser relativamente fácil cuando se trata de productos específicos para usuarios bien definidos, como los aeronáuticos, pero se complica cuando se trata de productos de uso general, como los avisos, que deben ser útiles y bien comprendidos por un público con muy variados intereses y capacidades, desde las autoridades, los responsables de emergencias y los medios de comunicación hasta el ciudadano de a pie.

Actualmente, además, la sociedad dispone con facilidad de información procedente de muy distintas fuentes, elaborada con distintos criterios y a veces distintos niveles de rigor, lo que en ocasiones puede llevar a confusión. Por otra parte, las redes sociales y los medios de comunicación nos permiten acercar nuestra información al ciudadano usando un lenguaje y unos recursos más versátiles y creativos. 


\section{PREDICCIÓN VS. PRODUCTOS}

La predicción meteorológica es, según la definición del Meteoglosario Visual de AEMET, el «anuncio o estimación, basado en las leyes físicas, del estado futuro de la atmósfera en un lugar y momento determinados». Es decir, todo el complejo proceso que se representa en la figura 1 no termina, como pudiéramos estar tentados de pensar en ocasiones, cuando hemos tomado y procesado todos los datos, aplicado nuestros cálculos y obtenido unos resultados, sino que el proceso tiene que alcanzar a unos destinatarios, que deben recibir un anuncio del estado futuro de la atmósfera adaptado a sus necesidades y capacidades.

\section{EL DESTINO DE LA PREDICCIÓN: EL USUARIO}

Puesto que hemos acordado que una predicción es un anuncio, y un anuncio no se concibe sin un destinatario, cuando le damos forma, es decir, cuando diseñamos y desarrollamos los productos NO debemos orientarlos al consumo interno, ni tomar en cuenta exclusivamente nuestros criterios, sino que nuestros productos de predicción deben estar siempre orientados a la medida de las necesidades y capacidades de los usuarios.

Esto puede ser relativamente fácil cuando se trata de productos específicos para usuarios bien definidos, como los aeronáuticos (figura 2), pero se complica cuando se trata de productos de uso general, como los avisos, que deben ser útiles y bien comprendidos desde las autoridades, los responsables de emergencias, los medios de comunicación y el ciudadano de a pie, con muy variados intereses y capacidades.

Es sabido que la mejor predicción del mundo no sirve para nada si no llega al alcance de los interesados, y además es bien comprendida. Finalmente —y esto es particularmente relevante en el caso de los avisos_- el destinatario debe saber cómo actuar en función de la predicción, por eso es importante la coordinación con las autoridades de protección civil responsables de las llamadas a la acción. El complemento perfecto a un aviso de un peligro meteorológico son las indicaciones de qué hacer al respecto.

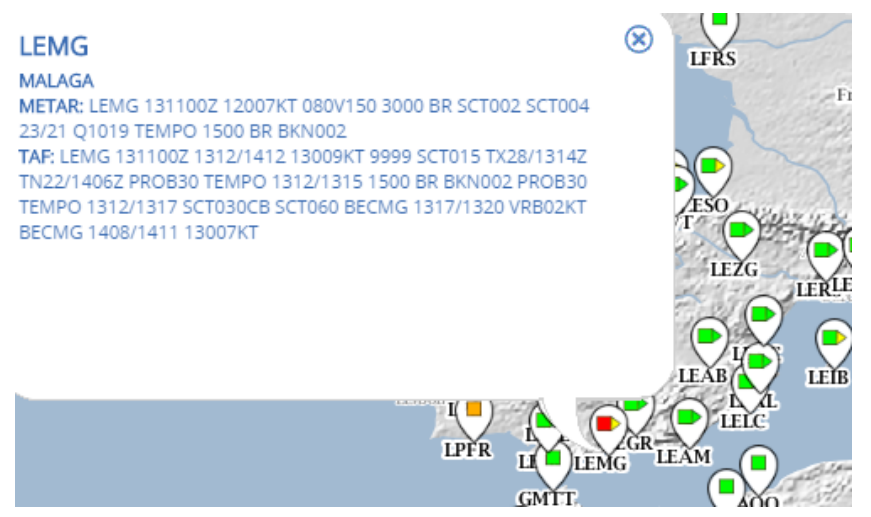

Figura 2.

Ejemplo de mensajes aeronáuticos cifrados en las claves internacionales METAR y TAF. 


\section{NUESTRO PAPEL FRENTE A LA DIVERSIDAD DE FUENTES DE INFORMACIÓN METEOROLÓGICA}

Hoy en día debemos tener bien presente que los usuarios tienen a su alcance mucha información meteorológica de muy diversas fuentes. Numerosas instituciones, empresas comerciales, aficionados y medios de comunicación difunden información de muy diversa índole, y elaborada con diversos criterios y niveles de rigor. Aunque la presentación y el formato a menudo son muy novedosos y creativos, no siempre se documentan suficientemente las características del producto mostrado, y a veces no se tienen en cuenta las limitaciones físicas propias del sistema atmosférico, que condicionan de forma inexorable la precisión del resultado (figura 3).

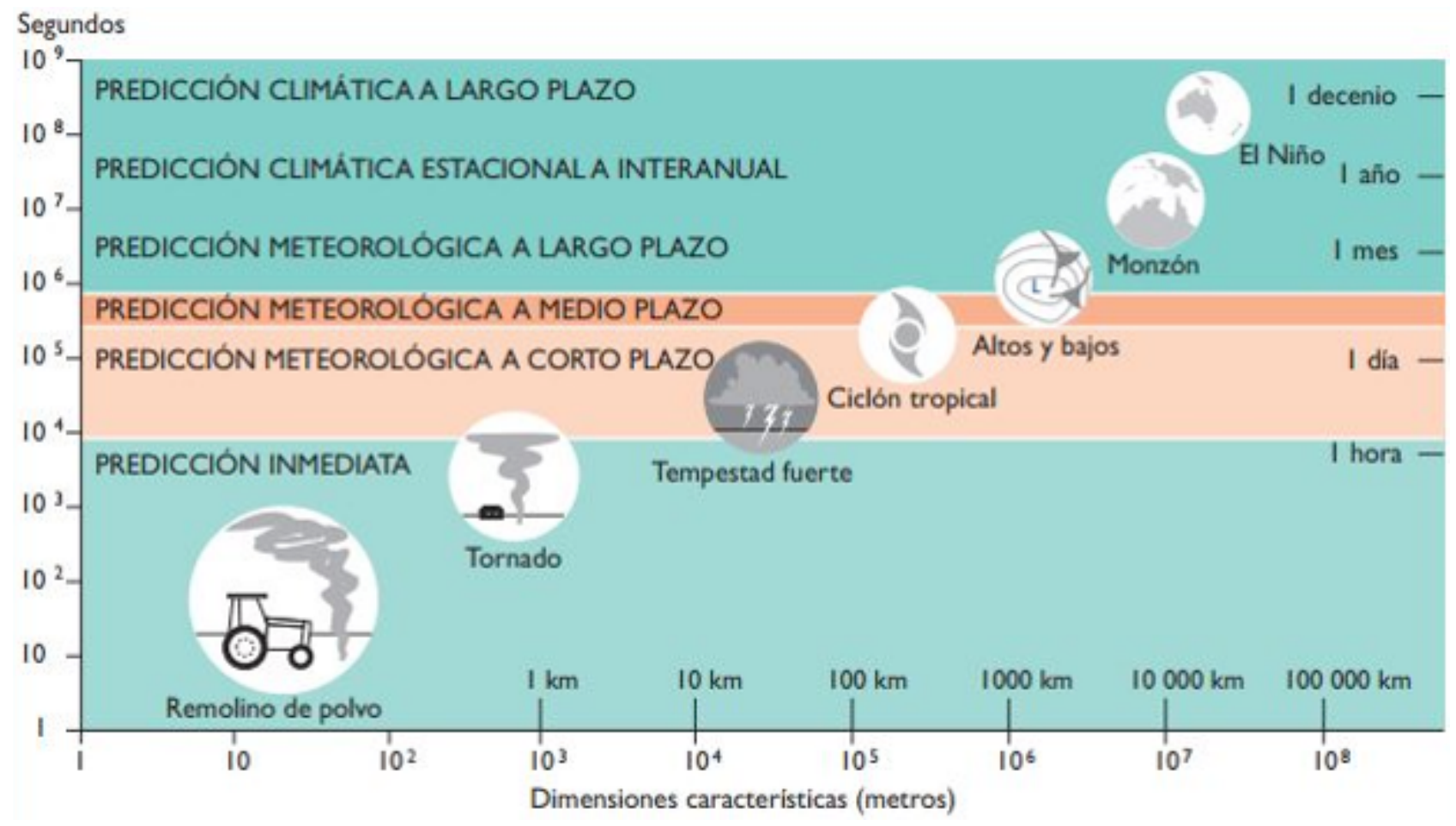

Figura 3. Escalas meteorológicas.

Fuente: Prevención de los desastres naturales y atenuación de sus efectos, OMM n. ${ }^{\circ} 993$.

Ante esta situación, debemos asumir la diversidad de información que el usuario tendrá a su alcance, pero también esforzarnos en dos sentidos. En primer lugar, en no quedarnos atrás en cuanto al diseño de nuestros formatos, para que nuestros productos no dejen de ser cómodos, eficientes y atractivos. En segundo lugar, y seguramente aún más importante, nuestros productos deben caracterizarse por estar muy bien documentados, por ser rigurosos, y por trasladar la incertidumbre asociada a las predicciones de manera comprensible.

Figura 4.

Serie integrada de predicciones.

Fuente: Guía de prácticas de servicios meteorológicos para el público, $\mathrm{OMM} \mathrm{n.}{ }^{\circ} 834$.

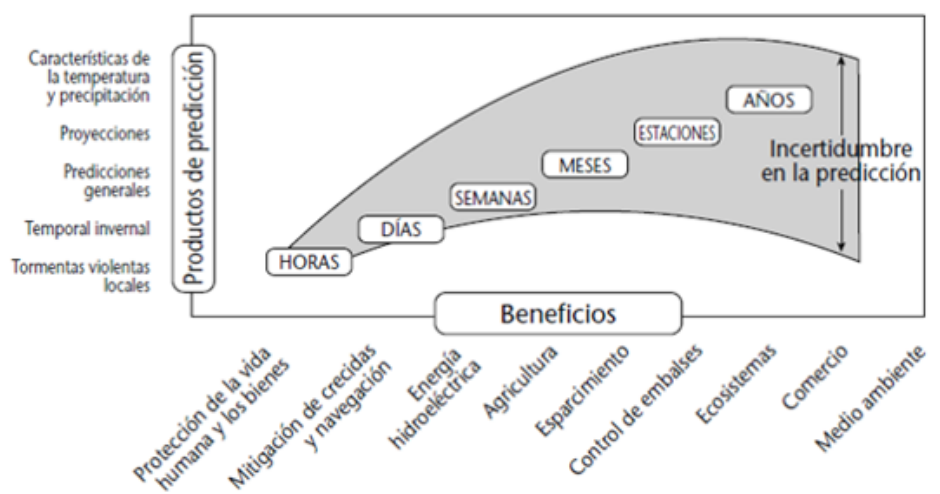




\section{VERSATILIDAD DE LAS REDES SOCIALES Y LOS MEDIOS DE COMUNICACIÓN}

Las redes sociales y los medios de comunicación son un complemento que nos permite difundir nuestros productos, darles visibilidad y acercarlos al ciudadano usando un lenguaje y unos recursos más versátiles y creativos.

Por ejemplo, a través de Twitter y Facebook podemos divulgar nuestras notas informativas y predicciones especiales, lo que resulta particularmente eficaz cuando hay mucha expectación. El uso de etiquetas o hashtags, de iconos, de imágenes ilustrativas, etc., enriquece el mensaje y lo hace más popular. Particularmente, las narraciones divulgativas en formato de vídeo muy breve están teniendo una gran acogida. También las retransmisiones en directo de eventos mediante el sistema Periscope, que satisfacen la curiosidad de cierto público interesado por la meteorología.

El canal de YouTube permite, mediante vídeos más largos, hacer divulgación, promocionar nuestras actividades, y dejar al alcance de los usuarios charlas, conferencias, etc.

El blog de AEMET es una herramienta que permite a cualquiera de los que componemos AEMET explayarnos de manera muy flexible, abierta y creativa, poniendo al alcance de los interesados información muy variada. Actualmente lo tenemos dividido en cinco secciones:

\section{AEMET EN ACCIÓN}

Información relacionada con actividades tales como eventos, cursos, jornadas, campañas, etc, realizadas por la Agencia Estatal de Meteorología.

\section{ENTENDER LA METEOROLOGÍA}

Textos técnicos y científicos sobre meteorología y climatología, e información meteorológica en formato divulgativo.

\section{PREDICCIONES ESPECÍFICAS}

Predicciones estacionales, semanales, boletines de aludes, etc., que no tienen una sección propia en el web institucional.

\section{RESÚMENES CLIMATOLÓGICOS}

Resúmenes climáticos e hidrológicos.

\section{ACTUALIDAD METEOROLÓGICA}

Resúmenes semanales del tiempo. Efemérides. Últimas noticias sobre el tiempo, el clima y la meteorología en España y el mundo.

No dejaremos de mencionar otras herramientas desarrolladas en AEMET, como el MeteoGlosario, que pone a disposición del público definiciones de los términos habituales en nuestros productos; SINOBAS, que recoge información ciudadana a la vez que difunde información que es particularmente bien acogida entre el mundillo interesado en la meteorología; MeteoRuta y MeteoNav, que facilitan nuestros avisos y nuestra información marítima en unos formatos específicamente dirigidos a conductores y a navegantes, respectivamente; o el repositorio Arcimís que ha puesto al alcance del público muchísima documentación. Este tipo de esfuerzos acercan nuestro trabajo a su destino real y le dan sentido. 


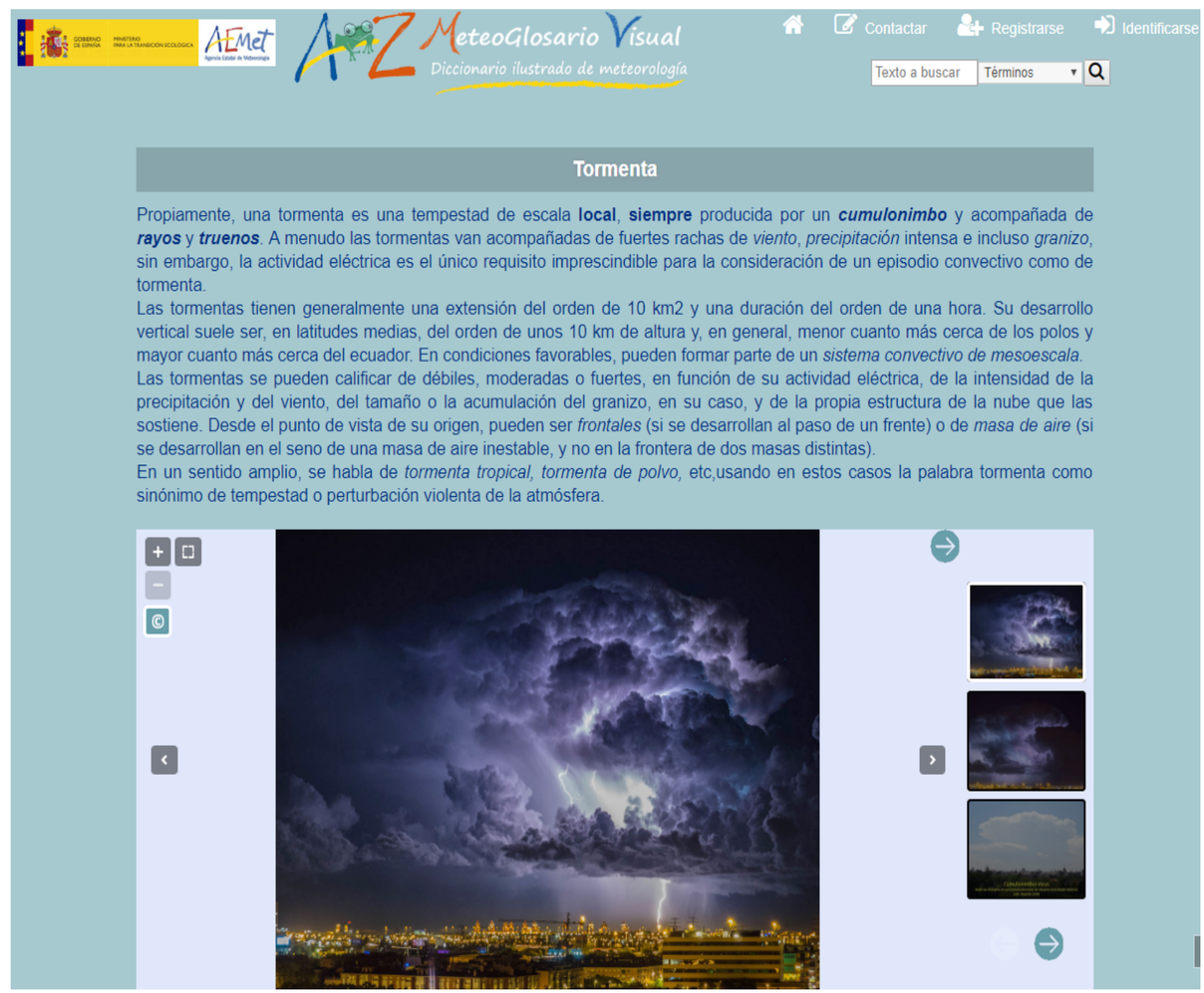

Figura 5. Ejemplo de definición en el MetoGlosario Visual de AEMET.

También el contacto asiduo con los medios de comunicación supone un recurso nada despreciable para dar a conocer nuestro trabajo y facilitar su correcta interpretación. Para un servicio meteorológico es una obligación, pero también una forma de afianzar nuestra imagen y nuestro prestigio, el responder de manera ágil y transparente a los requerimientos de los medios. La Organización Meteorológica Mundial, en su «Declaración sobre el papel y funcionamiento de los servicios meteorológicos e hidrológicos nacionales», destaca que «debido a que los medios de comunicación son un canal importante para transmitir al público las predicciones y los avisos, es importante forjar relaciones constructivas con los medios de información mundiales, regionales, nacionales y locales, ya sean electrónicos o impresos, para mejorar la prestación de servicios al público».

Hay ocasiones en que nuestras predicciones se miran con particular expectación. En esos casos, todos nuestros recursos para divulgar y dar a conocer nuestro trabajo son pocos. Las festividades señaladas, las olas de calor y de frío, los episodios de nevadas que afectan núcleos urbanos o vías de comunicación destacados, los ciclones tropicales y las borrascas profundas que se acercan a nuestro territorio, las danas mediterráneas que dan lugar a grandes cantidades de precipitación, etc. También, aunque no se trate de predicción, los récords climatológicos son igualmente un asunto de mucho interés. Y, por supuesto, el calentamiento global y el cambio climático son por desgracia actualidad desde hace mucho tiempo. Los estudios de atribución y de los impactos del cambio climático son, por tanto, una demanda de la sociedad que no debemos perder de vista. 


\section{CONCLUSIONES}

Como resumen, queremos aportar una serie de recomendaciones que considerar cuando se elaboran productos de predicción:

- Debemos esforzarnos en que nuestros productos estén siempre bien descritos y documentados, basados en conceptos sólidos y claros, y en usar un léxico tan preciso y simple como sea posible.

- La fluidez en la comunicación entre los productores de información y los que nos dedicamos a la comunicación es no solo muy valiosa, sino imprescindible.

- Es muy deseable constituir equipos multidisciplinares y creativos para la revisión y el diseño de los productos, así como para la divulgación necesaria para que sean bien entendidos.

- La asistencia a encuentros en foros de usuarios, de comunicadores, de aficionados, etc. es una valiosa fuente de información de retorno sobre nuestros productos, así como de nuevas ideas.

- Es muy útil prestar atención a lo que hacen otros organismos y productores de información, tanto nacionales como internacionales.

- Es recomendable ser cuidadosos con las malas traducciones (tormenta/temporal, severo/fuerte, ...), que pueden generar confusión.

\section{AGRADECIMIENTOS}

A los compañeros y compañeras que nos facilitan apoyo, datos, aclaraciones...

A quienes se fijan en nuestro trabajo y nos ayudan a mejorar.

A quienes elaboran productos y se toman en serio nuestras sugerencias.

A quienes escriben para el blog de AEMET.

A Trinidad Bueno, que lo sabe todo, y si no, se entera.

A Javier Martínez, que no hace más que inventar.

\section{REFERENCIAS}

Organización MeteorolóGica Mundial (OMM), 1999. Guía de prácticas de servicios meteorológicos para el público, OMM-N. ${ }^{\circ} 834$.

Organización MeteorolóGica Mundial (OMM), 2006. Prevención de los desastres naturales y atenuación de sus efectos, OMM-N. ${ }^{\circ} 993$.

Organización Meteorológica Mundial (OMM), 2007. El tiempo, el clima y los servicios hídricos al alcance de todos, OMM-N. ${ }^{\circ} 1024$.

MeteoGlosario Visual, https://meteoglosario.aemet.es/index.php.

SINOBAS, https://sinobas.aemet.es/index.php.

MeteoRuta, http://meteoruta.aemet.es/p_index.html.

MeteoNav, http://meteonav.aemet.es/MeteoNav/.

Arcimís, https://repositorio.aemet.es/. 